\title{
Platelet-to-lymphocyte ratio and neutrophil-to- lymphocyte ratio may reflect differences in PD and MSA-P neuroinflammation patterns
}

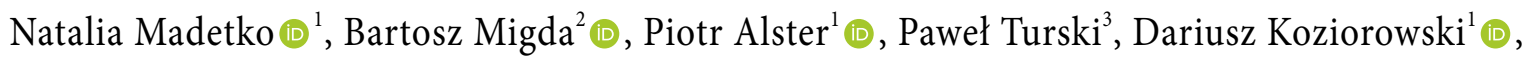 \\ Andrzej Friedman ${ }^{1}$ (D)
}

${ }^{1}$ Department of Neurology, Medical University of Warsaw, Poland

${ }^{2}$ Department of Paediatric Radiology, Medical University of Warsaw, Poland

${ }^{3}$ Students' Scientific Association of the Department of Neurology, Medical University of Warsaw, Poland

\begin{abstract}
Aim of the study. To assess the usefulness of neutrophil-to-lymphocyte ratio (NLR) and platelet-to-lymphocyte ratio (PLR) in evaluating the inflammatory process in alpha-synucleinopathies.

Clinical rationale for the study. The role of neuroinflammation in PD and MSA pathogenesis is indisputable. However, there is no method available in everyday use that would enable its evaluation. We suggest that NLR and PLR, as non-specific parameters of inflammation, due to its approachability could be helpful in the assessment of inflammatory activity in alpha-synucleinopathies in everyday clinical practice.
\end{abstract}

Material and methods. 98 patients with a clinical diagnosis of PD, 28 with MSA-P, and 99 healthy age-matched controls, were included in the study. Blood samples were analysed in order to count neutrophil and lymphocyte rates and, subsequently, NLR and PLR. The obtained parameters were compared between the groups. Results were statistically analysed.

Results. Our results indicate that patients with PD have higher values of NLR and PLR compared to controls. For MSA-P, only NLR was significantly higher in relation to the control group. There were no statistically significant differences between patients with PD and MSA-P in relation to NLR and PLR values. There was a positive average correlation between NLR and disease duration for MSA-P patients.

Conclusions. NLR and PLR values are significantly higher in alpha-synucleinopathies (MSA-P and PD) in relation to a control group. In PD patients, both NLR and PLR values are significantly higher in relation to a control group, whereas in patients with MSA-P, only NLR is significantly increased. The observed differences may reflect distinct neuroinflammatory patterns present in these entities.

Clinical implications. NLR and PLR are features of peripheral inflammation. Their specificity is relatively low, although increased values suggest possible inflammatory pathogenesis of clinical entities. NLR is based on the observations that in chronic and acute diseases the neutrophil rate has a tendency to rise, while the lymphocyte rate tends to decline. This aspect of inflammatory processes has been primarily evaluated in Intensive Care Units. PLR is a marker presenting changes in platelet and lymphocyte counts caused by acute inflammatory or prothrombotic states. Different values of NLR and PLR in PD and MSA-P compared to healthy controls suggest that in these two alpha-synucleinopathies, different patterns of neuroinflammation might be present. The role of inflammation in the differential diagnosis of parkinsonian syndromes remains unexplored.

Key words: PD, MSA-P, alpha-synucleinopathy, NLR, PLR, inflammation

(Neurol Neurochir Pol 2022; 56 (2): 148-155)

Address for correspondence: Natalia 1Madetko, Department of Neurology, Medical University of Warsaw, Kondratowicza Str. 8, 03-242 Warsaw, Poland; e-mail: natalia.madetko@wum.edu.pl

Received: 21.10.2021 Accepted: 27.12.2021 Early publication date: 4.02.2022

This article is available in open access under Creative Common Attribution-Non-Commercial-No Derivatives 4.0 International (CC BY-NC-ND 4.0) license, allowing to download articles and share them with others as long as they credit the authors and the publisher, but without permission to change them in any way or use them commercially. 


\section{Introduction}

Parkinson's Disease (PD) and Multiple System Atrophy (MSA) are progressive neurodegenerative disorders classified pathologically as alpha-synucleinopathies. PD was first described by James Parkinson in 1817 [1] and has been the subject of scientific interest ever since. Even so, the exact mechanism responsible for the process remains unclear. There are many theories concerning the pathogenesis of the disease. There is no doubt that mutations in several genes cause autosomal dominant or recessive forms of Parkinson's Disease. Many papers considering epigenetic abnormalities, exposure to toxins, oxidative stress, metabolic changes, telomere shortening, dysfunction of cellular proteolytic and mitochondrial system, or cardiovascular factors causing ischaemia, as potential causes of PD can be found in the literature [2-10]. Some studies have suggested that PD could be considered to be a prion-like disease [11]. Of all the discussed possible factors leading to PD pathology, the neuroinflammatory theory seems to be one of the most plausible.

It remains unclear, whether the prolonged inflammation in the cause or an effect of neurodegeneration, but the presence of this process is beyond doubt $[12,13]$. It has been stated that chronic neuroinflammatory process and microglial activation play crucial roles in the neurodegeneration observed in PD $[14,15]$. Chronic neuroinflammation can lead to bloodbrain-barrier damage that opens the door to central nervous system (CNS) infiltration by peripheral immune system cells and chemokines. This process can activate glial cells, T-cells and mast cells in the CNS, leading to increased neuroinflammation, which becomes chronic and results in neuronal loss. Mutual activation of inflammation in the CNS and peripheral immune cells leads to the release of neurotoxic molecules and exacerbates neurodegeneration [16]. Pro-inflammatory cytokines and chemokines prompt oxidative stress and damage to dopaminergic neurons [17].

Microglial activation seen as reactive oxygen species (ROS) synthesis is directly increased by $a$-synuclein, and this activity is even more severe in the case of mutated $\alpha$-synuclein forms compared to the wild type [18]. Moreover, microglialmediated inflammation (nuclear factor kappa-B and mitogenactivated protein kinase pathways) may be initiated by soluble $a$-synuclein when it binds to microglial TLR surface receptors $[19,20]$. Inflammation initiated in microglia causes the activation of astrocytes and, inter alia, the upregulation of nitric oxide (NO) production [21]. A high concentration of NO causes $\alpha$-synuclein aggregation [22] and promotes protein accumulation due to a decrease in proteasome activity [23]. This pathological cycle may be responsible for the prolonged neuroinflammation and progressive neurodegeneration seen in PD. In PD, neuroinflammation can be described as having a snowball-like effect with inflammatory activity gradually increasing over time.
MSA is often misdiagnosed as PD, especially the variant with predominant parkinsonism (MSA-P) and mainly in the early stages of the disease. Neuroinflammation is an important feature of MSA pathology, and can be observed as micro- and astrogliosis with increased proinflammatory cytokine levels [24]. Intensity of neuroinflammatory process is restricted to white matter regions due to the impact of oligodendrocytes containing $\alpha$-syn inclusions [24]. MSA animal models indicate that the inflammatory response is more intense compared to a PD model, which may suggest that increased inflammation in MSA from its early stages is responsible for its more aggressive clinical course [25].

Animal models of MSA indicate that neuroinflammation has an early, pre-symptomatic, onset with explicit response of myeloid cells with proliferative and phagocytic activity in areas with more pronounced alpha-synucleinopathy [24]. In later stages of the disease, proliferation and activity of myeloid cells decreases to a lesser but continuant proinflammatory level [24].

These findings indicate a specific pattern of neuroinflammation characterised by very severe inflammatory response at the onset of the disease. Widespread microglial activation in the early stages of MSA-P has also been described in humans [26]. Data concerning the intensity of peripheral inflammation in the course of MSA remain ambiguous [27, 28].

There are several methods of assessing neuroinflammation activity in vivo, featuring mainly neuroimaging with the use of specific radiotracers, e.g. $\left[{ }^{18} \mathrm{~F}\right]$-FEPPA PET [29] or 11CPK11195 PET [30]. However, these methods are not available in everyday clinical practice. Finding evidence for connections between neuroinflammation and peripheral inflammation may provide a solution to this challenge [31], as there is data supporting the interrelationship of the peripheral inflammatory response and neuroinflammation, meaning that the activity of one should reflect the activity of the other.

Neutrophil-to-lymphocyte ratio (NLR) is a parameter which was introduced in 2001 by Zahorec [32]. The value of NLR is calculated by dividing the number of neutrophils by the number of lymphocytes. The purpose of evaluating this parameter was to examine patients affected by systemic inflammation who were in a critical condition [32]. It was interpreted as a simple non-specific tool. Since the introduction of NLR, many researchers have verified the usefulness of this parameter in various diseases, not only those directly related to inflammatory involvement.

The role of NLR in Parkinson's Disease has been assessed in several papers; in the study by Moghaddam et al. [33], the authors verified an association between NLR and striatal binding ratios in DaT SPECT. The study showed that increased NLR is concomitant with a decrease in the striatal binding ratio and more pronounced motor impairment [33]. One of the studies interpreted NLR to be a possible marker of peripheral neuroinflammation in differentiating PD from progressive supranuclear palsy (PSP) [34]. The increase of NLR in PD has also been associated with a loss of neural connections [35]. The 
abnormalities were observed within cingulum bilaterally, body and left crus of fornix and corticospinal tract bilaterally [35]. An earlier work was based on the comparison of NLR in two variants of $\mathrm{PD}$ - the akinetic-rigid and the tremor-dominant [36]. This did not reveal any significant differences. To date, no study has evaluated NLR in the context of MSA.

Platelet-to-lymphocyte ratio (PLR) is calculated by dividing the number of platelets by the number of lymphocytes obtained from the same blood sample. As platelets are involved in peripheral inflammatory response, PLR is a parameter reflecting the level of inflammation. In the literature, this has mainly been discussed in the context of cardiovascular events [37], rheumatic diseases [38], cancer [39], and mental disorders [40]. This parameter has been assessed as a potential tool in differentiating PD from essential tremor [41], but there have been no papers considering this parameter in the context of MSA.

The aim of this study was to assess the intensity of peripheral inflammation in PD and MSA-P compared to healthy controls. We decided to use non-specific parameters, i.e. NLR and PLR, in order to evaluate the usefulness of tools available in everyday clinical practice.

\section{Clinical rationale for the study}

Neuroinflammation as an important factor involved in PD and MSA pathogenesis has been widely discussed in recent literature. It is possible that different patterns of ongoing neuroinflammatory process are responsible for its different clinical course and prognosis. However, the methods used in research facilities for neuroinflammation evaluation are not commonly available in everyday clinical practice.

The aim of this study was to assess whether NLR and PLR, as non-specific widespread parameters reflecting peripheral inflammation, could contribute to the diagnosis of PD/MSA and describe differences of inflammation intensity in $\mathrm{PD} /$ MSA and healthy age-matched populations. The results of our study could introduce a feasible tool into clinical practice, and generate a discussion regarding a possible correlation between, and the implications of, central and peripheral inflammation in the pathogenesis of alpha-synucleinopathies.

\section{Material and methods}

This study was based on a retrospective analysis of blood samples taken from patients with a clinical diagnosis of either Parkinson's Disease or Multiple System Atrophy who were hospitalised in the Department of Neurology at the Medical University of Warsaw, Poland. Diagnoses were made according to the current criteria $[42,43]$. The results of the control group were based on the routine examination of blood samples taken in the University's Department of Occupational Medicine. This study was approved by the Ethics Committee of Warsaw Medical University (AKBE151/2020).
Study participants did not suffer from any condition that could affect peripheral inflammation or blood count. The study included 98 patients with PD ( 43 females, 55 males, aged 39 to 85 years, mean 63 ), 28 patients with MSA-P ( 18 females, 10 males, aged 48 to 78 , mean 61), and 99 healthy controls (58 females, 41 males, aged 37 to 86, mean 57). Disease duration ranged from 1-6 years for MSA and 2-20 years for PD. All patients were treated with levodopa (medications combined with benserazide or carbidopa); daily dose ranged from 150 to $1,450 \mathrm{mg}$ for MSA patients (c. $850 \mathrm{mg}$ average) and from 400 to 2,100 mg for PD patients (c. 1,100 mg average). Less than 15\% of patients with PD included in the study received low doses of ropinirole - maximum $4 \mathrm{mg}$. No changes in drug dosage were made before taking the blood sample. The minimum duration of treatment at a fixed dose was 10 weeks. Other medications in the analysed population included: donepezil, low doses of pyridostigmine (due to constipation), levothyroxine, and metformin.

The exclusion criteria were: age under 35 , active infection, chronic inflammatory disease, neoplasm, haematopoietic abnormalities, drug use (including parkinsonian treatment) affecting blood count, diabetes, and significant cardiovascular disorders. Patient data was obtained by analysing their medical records. NLR and PLR ratios were compared between the groups.

The results were statistically analysed using the Shapiro-Wilk test, the Kruskal-Wallis ANOVA test with pairwise multiple comparison of mean ranks (PMCMR) in post-hoc analysis, and Spearman's correlation. $\mathrm{p}$ values $<0.05$ were considered to be statistically significant.

\section{Statistical analysis}

All calculations were performed using Statistica software (version 13.1 Dell. Inc. Statsoft). Data distribution was assessed with a Shapiro-Wilk test. All results were expressed as medians with interquartile range. As the analysed data did not have a normal distribution, for group comparison we used Kruskal-Wallis ANOVA. Pairwise multiple comparison of mean ranks (PMCMR) was used for post-hoc analysis. Spearman's correlation coefficient was used to check the dependence of potential changes in NLR and PLR with disease duration for patients with PD and MSA-P.

\section{Results}

The WBC levels were within the normal range for all the subjects analysed $\left(4-10^{\star} 10^{3} / \mathrm{ul}\right)$.

\section{Basic and subgroup analysis}

Median values with interquartile range (Q1-Q3) of assessed parameters for whole groups and subgroups are set out in Table 1. 


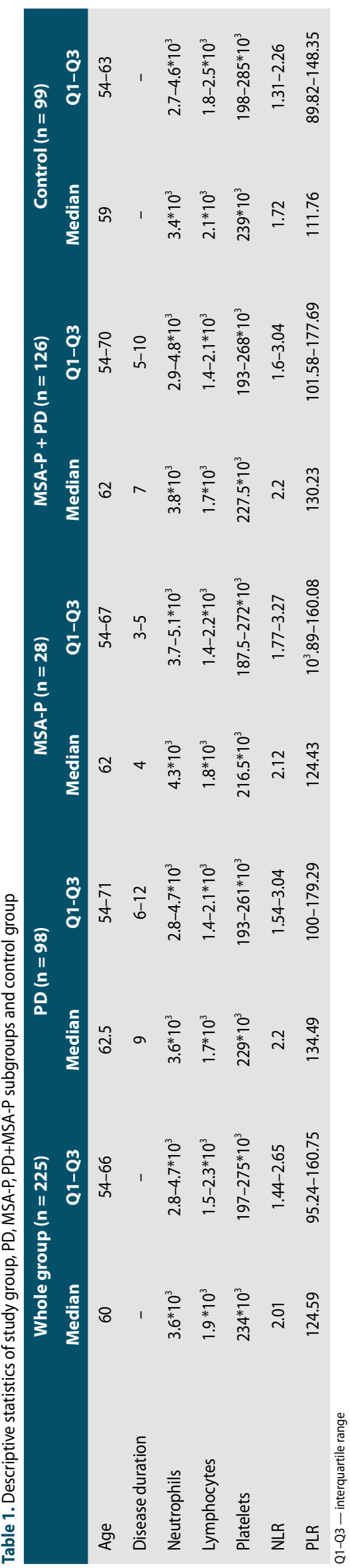

The PD patients had higher median values of NLR and PLR compared to controls, 2.2 vs 1.72 and 134.49 vs 111.76 , respectively ( $\mathrm{p}<0.05$, Tab. 2).

For MSA-P patients only, median of NLR was significantly higher in relation to the control group, 2.12 vs 1.72 ( $\mathrm{p}<0.05$, Tab. 2).

Unfortunately, there were no statistically significant differences between patients with PD and MSA-P in relation to NLR and PLR values.

\section{Spearman correlation}

There was a positive average correlation between NLR and disease duration for MSA-P patients $\mathrm{Rs}=0.5(\mathrm{p}<0.05, \mathrm{Tab} .3)$. For PD patients, Rs values were low Rs $<0.1$ ( $p>0.05$, Tab. 3 ).

\section{Discussion}

Studies looking into NLR as a potential parameter measuring inflammatory activity in PD have shown inconsistent results. Inci et al. [34] and Ataç Uçar et al. [36] reported no statistical difference in NLR values between PD and healthy controls, although Akıl et al. [44] observed a significantly higher NLR level in PD compared to a healthy population. This study proves that both NLR and PLR are significantly increased in a PD group compared to a control group.

The differences in the obtained results in the abovementioned studies might be explained by the size of the examined populations - the larger the studied group, the more pronounced were the differences in the NLR values observed. In a recently published paper analysing data obtained from $453 \mathrm{PD}$ patients and 436 controls, it was also proven that NLR is significantly higher in PD patients, although this paper did not describe PLR [45]. NLR has been found to correlate with white matter changes in PD [46], which suggests that it may, at least partially, reflect inflammatory and degenerative processes ongoing in the CNS. Some papers have proved that significantly increased peripheral inflammatory indices are correlated with the akinetic-rigid PD phenotype, whereas low peripheral inflammation markers are characteristic for patients with the tremor-dominant or mixed phenotypes [47].

In our study, increased NLR and PLR values distinguished PD and MSA-P from healthy controls and indicated the inflammatory process involved in disease pathogenesis, although for MSA-P patients, only the NLR value was sensitive enough. In MSA-P, the level of lymphocytes was not significantly higher than in PD. This resulted in the fact that the difference between the level of lymphocytes among MSA-P patients and the control group was much less pronounced than between PD and controls. This, combined with the relatively low number of platelets, was the cause of significant differences between PLR in PD patients and controls. This observation was not maintained in the comparison of MSA patients and controls. On the other hand, a high increase of neutrophils compared 
Table 2. Comparison of PD, MSA-P, and control groups

\begin{tabular}{|c|c|c|c|c|}
\hline & $\begin{array}{c}\text { PD vs MSA-P vs. control } \\
\qquad \text { p }^{*}\end{array}$ & $\begin{array}{c}\text { PD vs control } \\
\mathbf{p}^{* *}\end{array}$ & $\begin{array}{c}\text { MSA-P vs. control } \\
\text { p }^{* *}\end{array}$ & $\begin{array}{c}\text { PD vs. MSA-P } \\
\text { P }^{* *}\end{array}$ \\
\hline Age & 0.0041 & 0.0034 & 1.0000 & 0.3079 \\
\hline Neutrophils & 0.1047 & 0.9812 & 0.1064 & 0.4420 \\
\hline Lymphocytes & $<0.001$ & $<0.001$ & 0.0487 & 1.0000 \\
\hline Platelets & 0.2990 & 0.5719 & 0.6631 & 1.0000 \\
\hline NLR & $<0.001$ & $<0.001$ & 0.0176 & 1.0000 \\
\hline PLR & 0.0127 & 0.0105 & 0.4868 & 1.0000 \\
\hline
\end{tabular}

$p^{*}-p$-value for Kruskal-Wallis ANOVA; $p^{* *}-p$-value for pairwise multiple comparison of mean ranks (post-hoc test); in red are marked statistically significant $p$-values $<0.05$

Table 3. Correlations between PD, MSA-P, and disease duration

\begin{tabular}{lcc} 
& $\begin{array}{c}\text { PD }(\mathbf{n}=\mathbf{9 8}) \\
\text { disease duration } \\
\text { Rs }\end{array}$ & $\begin{array}{c}\text { MSA-P }(\mathbf{n}=\mathbf{2 8}) \\
\text { disease duration } \\
\text { Rs }\end{array}$ \\
\hline Neutrophils & -0.17 & 0.47 \\
Lymphocytes & -0.11 & -0.01 \\
Platelets & 0.00 & 0.10 \\
NLR & -0.08 & 0.50 \\
PLR & 0.07 & -0.06
\end{tabular}

Rs - Spearman correlation coefficient; in red are marked Rs values with $p<0.05$

to controls resulted in the fact that the comparison of NLR between MSA-P and controls showed significant differences, whereas in PLR the significance of differences was not confirmed. The tendency towards increased levels of neutrophils in MSA-P may be influenced by the differences in neuroinflammatory pattern of PD and MSA. Based on previous studies regarding cytokine profiling in the prefrontal cortex of $\mathrm{PD}$ and MSA patients, it has been shown that increased mRNA levels of GSK3 $\beta$ are observed in MSA but not in PD [48]. GSK3 $\beta$ is a relevant factor in the inhibition of AMP-activated protein kinase (AMPK). GSK3 $\beta$ inhibition of AMPK is an enhancer of lipopolysaccharide inflammatory responses resulting in stimulation of neutrophils [49]. The role of platelets in neurodegeneration is currently being widely discussed in the literature; due to neurotransmitters like $\gamma$-aminobutyric acid (GABA), glutamate, serotonin, epinephrine, dopamine, and histamine which are present in platelets cytoplasm or exosomes, these cells are thought to act as messengers connecting the CNS to the peripheral environment [50]. It is known that platelets release serotonin when exposed to glycolipid structures specific for neurons and astrocytes' lipid rafts [51], and this phenomenon occurs as a response to blood-brainbarrier damage and promotes neuroinflammation, inter alia in neurodegeneration [52]. According to Rydbirk et al. [48], there are no statistically significant differences in PDGF levels between PD and MSA, and therefore a disparity in platelet number should not be expected.

Another possible explanation for partially similar results of NLR and PLR obtained from patients with PD and MSA-P may lie in different inflammatory patterns. Taking into account the pace of deterioration and the previously described inflammatory patterns, it is possible that analysed parameters are similar due to inflammation in MSA fading over the course of time and incompletely developed neuroinflammation in middle-stage PD.

We hypothesise that the curves showing the severity of inflammation over time run in opposite directions, only to converge in the intermediate period of the diseases. Initially, the severity of inflammation should be much higher in MSA, while in late-stage PD, the severity of inflammation should exceed that seen in late-stage MSA. However, our theory requires verification. This hypothesis was not directly confirmed by our results, but they were obtained with the use of non-specific parameters of inflammation, and recent research concerning this issue suggests a multicausal background to this phenomenon. Kouli et al. [53] proved that at the earliest stages of PD there is a reduction in terminally differentiated effector memory (TEMRA) lymphocyte T CD8+ population compared to healthy controls. A study by Csencsits-Smith et al. [54] revealed that in MSA the dynamics of neuroinflammation acceleration measured by levels of serum cytokine secretion is four times greater than in $\mathrm{PD}$, which could explain faster progression.

However, due to the fact that the neuroinflammatory processes present in the pathogenesis of both the diseases under discussion is complex and includes multiple variables, it is very difficult to exactly determine which components of the immune system play the dominant role. Increased parameters of peripheral inflammation may be used as one of the biomarkers of alpha-synucleinopathies, among others, in non-invasive assessments [55].

To the best of our knowledge, this is the first study to assess NLR or PLR in MSA.

The fundamental limitation of this study is its retrospective character, which precluded an assessment of genetic features of included patients or other markers of the inflammatory process. All patients included in the study remain alive, and therefore all diagnoses were made according to current diagnostic criteria without neuropathological confirmation. 
NLR and PLR are non-specific parameters, although their assessment could be useful in everyday clinical practice. The exact mechanism of a possible association between peripheral inflammation and neuroinflammation must be further explored.

Therefore, it seems crucial to search for more specific parameters reflecting neuroinflammatory intensity that are accessible in everyday use. This is a topic that requires further investigation.

\section{Conclusions}

NLR and PLR values are significantly higher for alpha-synucleinopathies (MSA-P and PD) compared to a control group.

In PD patients, both NLR and PLR values are significantly higher compared to a control group, whereas in patients with MSA-P only, NLR is significantly higher.

NLR and PLR values do not help differentiate PD from MSA-P patients.

NLR and PLR values suggest the presence of different patterns of ongoing inflammation in PD and MSA-P.

\section{Clinical implications/future directions}

This study has identified a possible role of the everyday use of laboratory tests in the clinical diagnosis of PD and MSA-P. Our obtained results contribute to the discussion considering neuroinflammation and its possible peripheral markers in the context of alpha-synucleinopathies. This study highlights the need to search for accessible tools facilitating the management and diagnosis of Parkinson's Disease and Multiple System Atrophy. To the best of our knowledge, this is the first paper evaluating PLR in the context of atypical parkinsonian syndrome; this parameter should be assessed in patients with atypical parkinsonian syndromes, as previously published studies have shown interesting results in the context of peripheral inflammation markers [56].

Conflicts of interest: None.

Funding: None.

\section{References}

1. 1.Parkinson J. An essay on the shaking palsy. 1817. J Neuropsychiatry Clin Neurosci. 2002; 14(2): 223-36; discussion 222, doi: 10.1176/ jnp.14.2.223, indexed in Pubmed: 11983801.

2. Cacabelos R, Carrera I, Fernández-Novoa L, et al. Parkinson's Disease: New solutions to old problems. EuroEspes J. 2017; 11: 74-96.

3. Rokad D, Ghaisas S, Harischandra DS, et al. Role of neurotoxicants and traumatic brain injury in $\alpha$-synuclein protein misfolding and aggregation. Brain Res Bull. 2017; 133: 60-70, doi: 10.1016/j.brainresbull.2016.12.003, indexed in Pubmed: 27993598.

4. Toledo JB, Arnold SE, Raible K, et al. Contribution of cerebrovascular disease in autopsy confirmed neurodegenerative disease cases in the National Alzheimer's Coordinating Centre. Brain. 2013; 136(Pt
9): 2697-2706, doi: 10.1093/brain/awt188, indexed in Pubmed: 23842566.

5. Irwin D, Grossman M, Weintraub D, et al. Neuropathological and genetic correlates of survival and dementia onset in synucleinopathies: a retrospective analysis. The Lancet Neurology. 2017; 16(1): 55-65, doi: 10.1016/s1474-4422(16)30291-5.

6. Wen KX, Miliç J, El-Khodor B, et al. The role of DNA methylation and histone modifications in neurodegenerative diseases: a systematic review. PLoS One. 2016; 11(12): e0167201, doi: 10.1371/journal. pone.0167201, indexed in Pubmed: 27973581.

7. Nussbaum RL. Genentics of synucleopathies. Cold Spring Harb Perspect Med. 2017; 8(6), doi: 10.1101/cshperspect.a024109, indexed in Pubmed: 28213435.

8. Lill C. Genetics of Parkinson's disease. Molecular and Cellular Probes. 2016; 30(6): 386-396, doi: 10.1016/j.mcp.2016.11.001.

9. Xie $\mathrm{Yi}$, Feng $\mathrm{H}$, Peng $\mathrm{S}$, et al. Association of plasma homocysteine, vitamin B12 and folate levels with cognitive function in Parkinson's disease: A meta-analysis. Neurosci Lett. 2017; 636: 190-195, doi: 10.1016/j.neulet.2016.11.007, indexed in Pubmed: 27840145.

10. Scheffold A, Holtman IR, Dieni S, et al. Telomere shortening leads to an acceleration of synucleinopathy and impaired microglia response in a genetic mouse model. Acta Neuropathol Commun. 2016; 4(1): 87, doi: 10.1186/s40478-016-0364-x, indexed in Pubmed: 27550225.

11. Olanow CW, Brundin P. Parkinson's disease and alpha synuclein: is Parkinson's disease a prion-like disorder? Mov Disord. 2013; 28(1): 31-40, doi: 10.1002/mds.25373, indexed in Pubmed: 23390095.

12. Gelders $G$, Baekelandt $V$, Van der Perren $A$. Linking neuroinflammation and neurodegeneration in Parkinson's disease. J Immunol Res. 2018; 2018: 4784268, doi: 10.1155/2018/4784268, indexed in Pubmed: 29850629.

13. Ho MS. Microglia in Parkinson's Disease. Adv Exp Med Biol. 2019; 1175: 335-353, doi: 10.1007/978-981-13-9913-8_13, indexed in Pubmed: 31583594.

14. Tansey MG, Goldberg MS. Neuroinflammation in Parkinson's disease: its role in neuronal death and implications for therapeutic intervention. Neurobiol Dis. 2010; 37(3): 510-518, doi: 10.1016/j. nbd.2009.11.004, indexed in Pubmed: 19913097.

15. McGeer PL, Itagaki S, Boyes BE, et al. Reactive microglia are positive for HLA-DR in the substantia nigra of Parkinson's and Alzheimer's disease brains. Neurology. 1988; 38(8): 1285-1291, doi: 10.1212/ wnl.38.8.1285, indexed in Pubmed: 3399080.

16. Kempuraj D, Thangavel R, Selvakumar GP, et al. Brain and peripheral atypical inflammatory mediators potentiate neuroinflammation and neurodegeneration. Front Cell Neurosci. 2017; 11: 216, doi: 10.3389/ fncel.2017.00216, indexed in Pubmed: 28790893.

17. Tiwari PC, Pal R. The potential role of neuroinflammation and transcription factors in Parkinson disease. Dialogues Clin Neurosci. 2017; 19(1): 71-80, indexed in Pubmed: 28566949.

18. Rocha EM, De Miranda B, Sanders LH. Alpha-synuclein: Pathology, mitochondrial dysfunction and neuroinflammation in Parkinson's disease. Neurobiol Dis. 2018; 109(Pt B): 249-257, doi: 10.1016/j. nbd.2017.04.004, indexed in Pubmed: 28400134.

19. Kim C, Lee HJ, Masliah E, et al. Non-cell-autonomous Neurotoxicity of $\alpha$-synuclein Through Microglial Toll-like Receptor 2. Experimental Neurobiology. 2016; 25(3): 113-119, doi: 10.5607/en.2016.25.3.113.

20. Wang $\mathrm{S}$, Chu $\mathrm{CH}$, Stewart T, et al. $\alpha$-Synuclein, a chemoattractant, directs microglial migration via H202-dependent Lyn phosphorylation. Proceedings of the National Academy of Sciences. 2015; 112(15): E1926-E1935, doi: 10.1073/pnas.1417883112. 
21. Hewett S, Corbett J, McDaniel M, et al. Interferon-y and interleukin-1 $\beta$ induce nitric oxide formation from primary mouse astrocytes. Neuroscience Letters. 1993; 164(1-2): 229-232, doi: 10.1016/03043940(93)90898-u.

22. Paxinou $E$, Chen $Q$, Weisse $M$, et al. Induction of $\alpha$-synuclein aggregation by intracellular nitrative insult. The Journal of Neuroscience. 2001; 21(20): 8053-8061, doi: 10.1523/jneurosci.21-20-08053.2001.

23. Gu Z, Nakamura T, Yao D, et al. Nitrosative and oxidative stress links dysfunctional ubiquitination to Parkinson's disease. Cell Death Differ. 2005; 12(9): 1202-1204, doi: 10.1038/sj.cdd.4401705, indexed in Pubmed: 16094397.

24. Hoffmann A, Ettle B, Battis K, et al. Oligodendroglial $\alpha$-synucleinopathydriven neuroinflammation in multiple system atrophy. Brain Pathol. 2019; 29(3): 380-396, doi: 10.1111/bpa.12678, indexed in Pubmed: 30444295.

25. Harms AS, Kordower JH, Sette A, et al. Inflammation in experimental models of $\alpha$-synucleinopathies. Mov Disord. 2021; 36(1): 37-49, doi: 10.1002/mds.28264, indexed in Pubmed: 33009855.

26. Kübler D, Wächter T, Cabanel N, et al. Widespread microglial activation in multiple system atrophy. Mov Disord. 2019; 34(4): 564-568, doi: 10.1002/mds.27620, indexed in Pubmed: 30726574.

27. Kim R, Kim HJ, Kim A, et al. Does peripheral inflammation contribute to multiple system atrophy? Parkinsonism Relat Disord. 2019; 64: 340-341, doi: 10.1016/j.parkreldis.2019.03.020, indexed in Pubmed: 30940429.

28. Kaufman E, Hall S, Surova Y, et al. Proinflammatory cytokines are elevated in serum of patients with multiple system atrophy. PLoS One. 2013; 8: e62354.

29. Ghadery C, Koshimori Y, Coakeley S, et al. Microglial activation in Parkinson's disease using [F]-FEPPA. J Neuroinflammation. 2017; 14(1): 8, doi: 10.1186/s12974-016-0778-1, indexed in Pubmed: 28086916.

30. Nicastro N, Surendranathan A, Mak E, et al. C-PK11195 PET imaging and white matter changes in Parkinson's disease dementia. Ann Clin Transl Neurol. 2019; 6(10): 2133-2136, doi: 10.1002/acn3.50877, indexed in Pubmed: 31507085.

31. Kustrimovic N, Marino F, Cosentino M, et al. Dopaminergic receptors on CD4+ T naive and memory lymphocytes correlate with motor impairment in patients with Parkinson's disease. Sci Rep. 2016; 6(1): 33738-3753, doi: 10.1038/srep33738, indexed in Pubmed: 27652978.

32. Zahorec R. Ratio of neutrophil to lymphocyte counts--rapid and simple parameter of systemic inflammation and stress in critically ill. Bratisl Lek Listy. 2001; 102(1): 5-14, indexed in Pubmed: 11723675.

33. Sanjari Moghaddam H, Ghazi Sherbaf F, Mojtahed Zadeh M, et al. Association between peripheral inflammation and DATSCAN data of the striatal nuclei in different motor subtypes of Parkinson disease. Front Neurol. 2018; 9: 234, doi: 10.3389/fneur.2018.00234, indexed in Pubmed: 29713303.

34. Inci I, Kusbeci OY, Eskut N. The neutrophil-to-lymphocyte ratio as a marker of peripheral inflammation in progressive supranuclear palsy: a retrospective study. Neurol Sci. 2020; 41(5): 1233-1237, doi: 10.1007/s10072-019-04208-4, indexed in Pubmed: 31901125.

35. Haghshomar M, Rahmani F, Hadi Aarabi M, et al. White matter changes correlates of peripheral neuroinflammation in patients with Parkinson's disease. Neuroscience. 2019; 403: 70-78, doi: 10.1016/j. neuroscience.2017.10.050, indexed in Pubmed: 29126955.

36. Ataç Uçar C, Gökçe Çokal B, Ünal Artık HA, et al. Comparison of neutrophil-lymphocyte ratio (NLR) in Parkinson's disease subtypes.
Neurol Sci. 2017; 38(2): 287-293, doi: 10.1007/s10072-016-27588, indexed in Pubmed: 27837368.

37. Balta S, Ozturk C. The platelet-lymphocyte ratio: A simple, inexpensive and rapid prognostic marker for cardiovascular events. Platelets. 2015; 26(7): 680-681, doi: 10.3109/09537104.2014.979340, indexed in Pubmed: 25549287.

38. Gasparyan AY, Ayvazyan L, Mukanova U, et al. The Platelet-to-Lymphocyte ratio as an inflammatory marker in rheumatic diseases. Ann Lab Med. 2019; 39(4): 345-357, doi: 10.3343/alm.2019.39.4.345, indexed in Pubmed: 30809980.

39. Li Bo, Zhou P, Liu Y, et al. Platelet-to-lymphocyte ratio in advanced cancer: review and meta-analysis. Clin Chim Acta. 2018; 483: 48-56, doi: 10.1016/j.cca.2018.04.023, indexed in Pubmed: 29678631.

40. Mazza MG, Lucchi S, Rossetti A, et al. Neutrophil-lymphocyte ratio, monocyte-lymphocyte ratio and platelet-lymphocyte ratio in non-affective psychosis: A meta-analysis and systematic review. World J Biol Psychiatry. 2020; 21(5): 326-338, doi: 10.1080/15622975.2019.1583371, indexed in Pubmed: 30806142.

41. Acar T, Aras YG, Acar BA. Can the neutrophil-lymphocyte ratio and platelet-lymphocyte ratio be used in the differential diagnosis of Parkinson's Disease and essential tremor? Acta Medica Mediterranea. 2019; 35: 929, doi: 10.19193/0393-6384_2019_2_141.

42. Postuma RB, Berg D, Stern M, et al. MDS clinical diagnostic criteria for Parkinson's disease. Mov Disord. 2015; 30(12): 1591-1601, doi: 10.1002/mds.26424, indexed in Pubmed: 26474316.

43. Gilman S, Wenning GK, Low PA, et al. Second consensus statement on the diagnosis of multiple system atrophy. Neurology. 2008; 71(9): 670-676, doi: 10.1212/01.wnl.0000324625.00404.15, indexed in Pubmed: 18725592.

44. Akıl E, Bulut A, Kaplan I, et al. The increase of carcinoembryonic antigen (CEA), high-sensitivity C-reactive protein, and neutrophil/lymphocyte ratio in Parkinson's disease. Neurol Sci. 2015; 36(3): 423-428, doi: 10.1007/s10072-014-1976-1, indexed in Pubmed: 25288159.

45. Wang Y, Gao H, Jiang S, et al. Principal component analysis of routine blood test results with Parkinson's disease: A case-control study. Exp Gerontol. 2021; 144: 111188, doi: 10.1016/j.exger.2020.111188, indexed in Pubmed: 33279667.

46. Haghshomar M, Rahmani F, Hadi Aarabi M, et al. White matter changes correlates of peripheral neuroinflammation in patients with Parkinson's disease. Neuroscience. 2019; 403: 70-78, doi: 10.1016/j. neuroscience.2017.10.050, indexed in Pubmed: 29126955.

47. Umehara T, Oka H, Nakahara A, et al. Differential leukocyte count is associated with clinical phenotype in Parkinson's disease. J Neurol Sci. 2020; 409: 116638, doi: 10.1016/j.jns.2019.116638, indexed in Pubmed: 31865186.

48. Rydbirk R, Elfving B, Andersen MD, et al. Cytokine profiling in the prefrontal cortex of Parkinson's Disease and Multiple System Atrophy patients. Neurobiol Dis. 2017; 106: 269-278, doi: 10.1016/j. nbd.2017.07.014, indexed in Pubmed: 28732710.

49. Park DW, Jiang S, Liu Y, et al. GSK3ß-dependent inhibition of AMPK potentiates activation of neutrophils and macrophages and enhances severity of acute lung injury. Am J Physiol Lung Cell Mol Physiol. 2014; 307(10): L735-L745, doi: 10.1152/ajplung.00165.2014, indexed in Pubmed: 25239914.

50. Leiter 0 , Walker TL. Platelets in neurodegenerative conditionsfriend or foe? Front Immunol. 2020; 11: 747, doi: 10.3389/fimmu.2020.00747, indexed in Pubmed: 32431701.

51. Sotnikov I, Veremeyko T, Starossom SC, et al. Platelets recognize brain-specific glycolipid structures, respond to neurovascular damage 
and promote neuroinflammation. PLoS One. 2013; 8(3): e58979, doi: 10.1371/journal.pone.0058979, indexed in Pubmed: 23555611.

52. Carvey PM, Hendey B, Monahan AJ. The blood-brain barrier in neurodegenerative disease: a rhetorical perspective. J Neurochem. 2009; 111(2): 291-314, doi: 10.1111/j.1471-4159.2009.06319.x, indexed in Pubmed: 19659460.

53. Kouli A, Jensen M, Papastavrou V, et al. T lymphocyte senescence is attenuated in Parkinson's disease. J Neuroinflammation. 2021; 18(1): 228, doi: 10.1186/s12974-021-02287-9, indexed in Pubmed: 34645462.

54. Csencsits-Smith K, Suescun J, Li K, et al. Serum lymphocyte-associated cytokine concentrations change more rapidly over time in multiple system atrophy compared to Parkinson disease. Neuroimmunomodulation. 2016; 23(5-6): 301-308, doi: 10.1159/000460297, indexed in Pubmed: 28395279.

55. Figura M, Friedman A. In search of Parkinson's disease biomarkers - is the answer in our mouths? A systematic review of the literature on salivary biomarkers of Parkinson's disease. Neurol Neurochir Pol. 2020; 54(1): 14-20, doi: 10.5603/PJNNS.a2020.0011, indexed in Pubmed: 32003440.

56. Alster P, Madetko N, Friedman A. Neutrophil-to-lymphocyte ratio (NLR) at boundaries of Progressive Supranuclear Palsy Syndrome (PSPS) and Corticobasal Syndrome (CBS). Neurol Neurochir Pol. 2021; 55(1): 97-101, doi: 10.5603/PJNNS.a2020.0097, indexed in Pubmed: 33315235. 